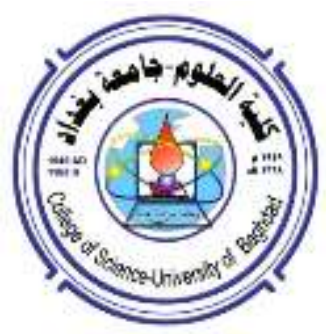

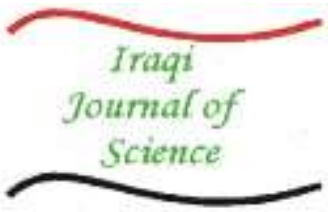

ISSN: 0067-2904

\title{
Quasi J-Regular Modules
}

\author{
Rafid M. AL - Shaiban Nuhad S. AL-Mothafar \\ Department of Mathematic, College of Science, University of Baghdad, Baghdad, Iraq
}

Received: 17/9/2019 Accepted: 31/10/2019

\begin{abstract}
Throughout this note, $\mathrm{R}$ is commutative ring with identity and $\mathrm{M}$ is a unitary $\mathrm{R}$ module. In this paper, we introduce the concept of quasi J-pure submodules as a generalization of quasi-pure submodules and give some of its basic properties. Using this concept, we define the class of quasi J-regular modules, where an Rmodule $\mathrm{M}$ is called quasi J-regular module if every submodule of $\mathrm{M}$ is quasi J-pure. Many results about this concept are proved.
\end{abstract}

Keywords: J-pure submodules, quasi J-pure submodules, J-regular modules and quasi J-regular modules.

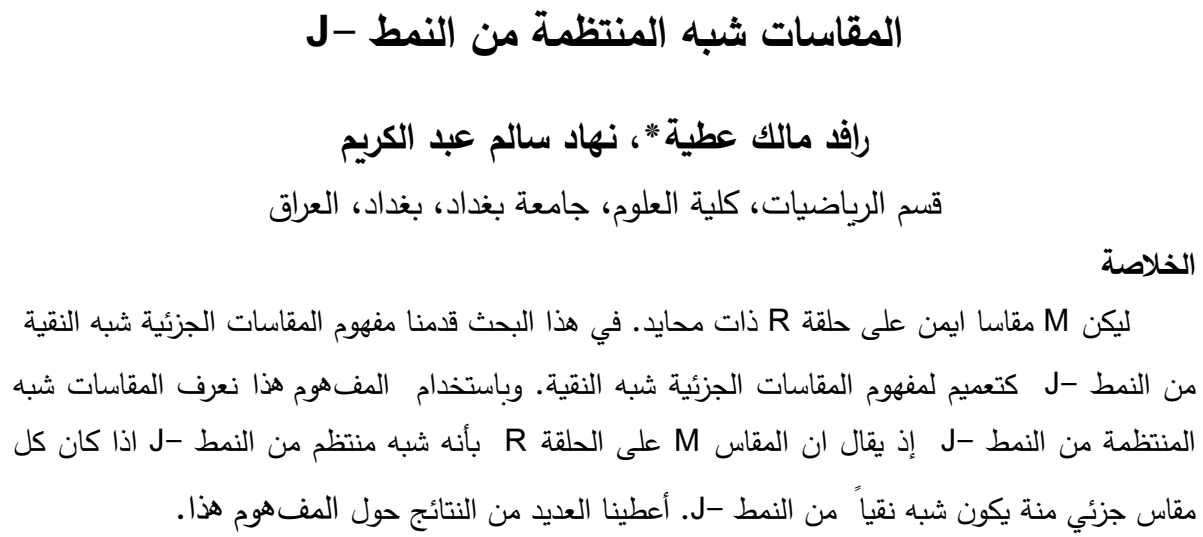

\section{Introduction}

$\mathrm{M}$ is viewed as a right module over an arbitrary ring with identity. A submodule $\mathrm{N}$ of an $\mathrm{R}$-module $\mathrm{M}$ is called pure in $\mathrm{M}$ if IN = N $\cap$ IM for every ideal I of $\mathrm{R}$ [1]. An R-module $\mathrm{M}$ is a regular module if every submodule of $\mathrm{M}$ is pure [2]. A submodule $\mathrm{N}$ of an $\mathrm{R}$-module $\mathrm{M}$ is called a $\mathrm{J}$-pure if $\mathrm{N}$ is pure in $J(M)$, i.e. for each ideal I of R, I J (M) N = IN, where J (M) is the Jacobson radical of M. An Rmodule $\mathrm{M}$ is said to be $\mathrm{J}$-regular module if every submodule of $\mathrm{M}$ is J-pure submodule. Equivalently, an R-module $\mathrm{M}$ is said to be J-regular module if for each $m \in \mathrm{J}(\mathrm{M}), r \in \mathrm{R}$, there exists $t \in$ Rsuch that $r m=r t r$ [3].

First, recall that a submodule $\mathrm{N}$ of an $\mathrm{R}$-module $\mathrm{M}$ is called a quasi - pure if, for each $x \in \mathrm{M}$ and $x \notin \mathrm{N}$, there exists a pure submodule $\mathrm{L}$ of $\mathrm{M}$ such that $\mathrm{N} \subseteq \mathrm{L}$ and $x \notin \mathrm{L}$, and an $\mathrm{R}$-module $\mathrm{M}$ is called quasi - regular module if every submodule of $\mathrm{M}$ is quasi - pure [4]. This paper is structured in two sections. In section one we introduce a comprehensive study of J-pure submodules. Some results are analogous to the properties of pure submodules. In section two, we study the concept of quasi Jregular modules.

\section{Quasi J-pure Submodules}

In this section we introduce the concept of quasi J-pure submodule. We investigate the basic properties of these types of submodules which are analogous to the properties of J-pure submodules. 


\section{Definition (2.1):}

Let $\mathrm{M}$ be an R-module. A submodule $\mathrm{N}$ of $\mathrm{M}$ is called a quasi J-pure submodule of $\mathrm{M}$ if for each $x \in \mathrm{M}$ and $x \notin \mathrm{N}$, there exists a $\mathrm{J}$-pure submodule $\mathrm{L}$ of $\mathrm{M}$ such that $\mathrm{N} \subseteq \mathrm{L}$ and $x \notin \mathrm{L}$.

\section{Remarks and Examples (2.2)}

(1) It is clear that every J-pure submodule is quasi J-pure. But the converse is not true in general. For example, let $\mathrm{M}=Z_{8} \oplus Z_{2}$ be a Z-module, and $\mathrm{N}=\langle(\overline{4}, \overline{0})\rangle=\{(\overline{0}, \overline{0}),(\overline{4}, \overline{0})\}$. It is easily checked that $\mathrm{N}$ is quasi $\mathrm{J}$-pure submodule of $\mathrm{M}$, since for each $x \in \mathrm{M}$ and $x \notin \mathrm{N}$, there exists a J-pure submodule $\mathrm{L}$ of $\mathrm{M}$ containing $\mathrm{N}$ and $x \notin \mathrm{L}$. But $\mathrm{N}$ is not $\mathrm{J}$-pure submodule of $\mathrm{M}$, since $(\overline{4}, \overline{0})=$ $2(\overline{2}, \overline{0}) \in 2 \mathrm{~J}\left(Z_{8} \oplus Z_{2}\right) \cap N$, but $(\overline{4}, \overline{0}) \notin 2 . N=\{(\overline{0}, \overline{0})\}$.

(2) In any R-module M, the submodule $\langle 0\rangle$ is always quasi J-pure.

(3) It is clear that every quasi -pure is quasi J-pure but the converse is not true. For example, the submodule $\{\overline{0}, \overline{2}\}$ in the Z-module $Z_{4}$ is quasi J-pure. Since it is J-pure, but $\{\overline{0}, \overline{2}\}$ is not quasi - pure. Since there exists no pure submodule that contains $\{\overline{0}, \overline{2}\}$.

Recall that an R-module $\mathrm{M}$ is called $\mathrm{J}$-pure simple if $\mathrm{M}$ and $\langle 0\rangle$ are the only $\mathrm{J}$-pure submodules of $\mathrm{M}$.

(4) Every J-pure simple R-module $\mathrm{M}$ does not contain quasi J-pure submodule except $\langle 0\rangle$ and $\mathrm{M}$. For example, the $\mathrm{Z}$-modules $\mathrm{Q}, \mathrm{Z}_{\mathrm{P}^{\infty}}$. $\mathrm{Q}$ as $\mathrm{Z}$-module is J-pure simple, hence $\mathrm{Q}$ does not have $\mathrm{J}$-pure submodule except $\langle 0\rangle$ and $\mathrm{Q}$. Since $\mathrm{Q} \cap \mathrm{IJ}(\mathrm{Q})=\mathrm{Q} \cap \mathrm{IQ}=\mathrm{IQ}$, then $\mathrm{Q}$ is quasi J-pure since it is $\mathrm{J}$ pure.

(5) If $\mathrm{N}_{1}$ and $\mathrm{N}_{2}$ are quasi J-pure submodules of an R-module $\mathrm{M}$, then $\mathrm{N}_{1} \cap \mathrm{N}_{2}$ is quasi Jpure submodule of $\mathrm{M}$. To show this, let $x \in \mathrm{M}$ and $x \notin \mathrm{N}_{1} \cap \mathrm{N}_{2}$, then either $x \notin \mathrm{N}_{1}$ or $x \notin \mathrm{N}_{2}$. If $x \notin \mathrm{N}_{1}$, since $\mathrm{N}_{1}$ is quasi J-pure in $\mathrm{M}$, then there exists a J-pure submodule $\mathrm{L}$ of $\mathrm{M}$ such that $\mathrm{N}_{1} \subseteq \mathrm{L}$ and $x \notin \mathrm{L}$. Hence $\mathrm{N}_{1} \cap \mathrm{N}_{2} \subseteq \mathrm{L}$. Similarly if $x \notin \mathrm{N}_{2}$.

(6) The sum of two quasi J-pure submodules may not be quasi J-pure. For example, consider the Zmodule $\mathrm{M}=Z_{8} \oplus Z_{2}$. Let $\mathrm{N}=\langle(\overline{4}, \overline{1})>$ and $\mathrm{L}=\langle(\overline{2}, \overline{0})>$. It is easy to see that $\mathrm{N}$ and $\mathrm{L}$ are quasi pure submodules in M. But $\mathrm{N}+\mathrm{L}=\{(\overline{0}, \overline{0}),(\overline{2}, \overline{0}),(\overline{4}, \overline{0}),(\overline{6}, \overline{0}),(\overline{4}, \overline{1})\}$ is not quasi J-pure submodule in $M$.

The following propositions give some properties of quasi J-pure submodules.

\section{Proposition (2.3)}

Let $\mathrm{M}$ be an R-module and $\mathrm{N}$ be a J-pure submodule of $\mathrm{M}$. If $\mathrm{B}$ is a quasi J-pure submodule of $\mathrm{N}$, then B is a quasi J-pure submodule of M.

\section{Proof}

Let $x \in \mathrm{M}$ with $x \notin \mathrm{B}$, then either $x \in \mathrm{N}$ or $x \notin \mathrm{N}$. Assume that $x \in \mathrm{N}$, but $\mathrm{B}$ is a quasi J-pure submodule in $\mathrm{N}$, so there exists a J-pure submodule $\mathrm{L}$ in $\mathrm{N}$ such that $\mathrm{B} \subseteq \mathrm{L}$ and $x \notin \mathrm{L}$. Thus we have $\mathrm{L}$ is $\mathrm{J}$-pure in $\mathrm{N}$ and $\mathrm{N}$ is $\mathrm{J}$-pure in $\mathrm{M}$, so by 3, remark 2.3, L is J-pure in $\mathrm{M}$. Therefore, B is quasi Jpure submodule of M. Now, if $x \notin \mathrm{N}$, then there is nothing to prove, since $\mathrm{N}$ is a J-pure submodule in $\mathrm{M}$ containing B and $x \notin \mathrm{N}$.

Proposition (2.4)

Let $\mathrm{R}$ be a good ring, $\mathrm{M}$ be an R-module and $\mathrm{N}$ be a J-pure submodule of $\mathrm{M}$. If $\mathrm{B}$ is a submodule of $\mathrm{M}$ containing $\mathrm{N}$, then $\mathrm{N}$ is a quasi $\mathrm{J}$-pure submodule of $\mathrm{B}$.

\section{Proof}

Since $\mathrm{N}$ is J-pure submodule of $\mathrm{M}$ and $\mathrm{N} \subseteq \mathrm{B}$, since $\mathrm{R}$ is a good ring. So, as previously shown [3, proposition 2.4], $\mathrm{N}$ is a $\mathrm{J}$-pure submodule of $\mathrm{B}$, which implies that $\mathrm{N}$ is quasi $\mathrm{J}$-pure submodule of $\mathrm{B}$.

Recall that the A submodule $N$ of an $R$ - module $\mathrm{M}$ is called a small submodule of $\mathrm{M}$ (notation $N<<\mathrm{M}$ ), if for any submodule $A$ of $\mathrm{M}$ such that $\mathrm{M}=N+A$, then $A=\mathrm{M}$ [5].

\section{Proposition (2.5)}

Let $\mathrm{M}$ be an R-module and $\mathrm{N}$ be a quasi J-pure submodule of $\mathrm{M}$. If $\mathrm{H}$ is a small submodule of $\mathrm{N}$, then $\frac{\mathrm{N}}{\mathrm{H}}$ is a quasi J-pure submodule of $\frac{\mathrm{M}}{\mathrm{H}}$.

\section{Proof}

Let $x+\mathrm{H} \in \frac{\mathrm{M}}{\mathrm{H}}$ with $x+\mathrm{H} \notin \frac{\mathrm{N}}{\mathrm{H}}$. Then $x \notin \mathrm{N}$ and $x \in \mathrm{M}$. But $\mathrm{N}$ is quasi J-pure in M. So there exists a J-pure submodule $\mathrm{L}$ of $\mathrm{M}$ such that $\mathrm{N} \subseteq \mathrm{L}$ and $x \notin \mathrm{L}$. This implies that $\frac{\mathrm{N}}{\mathrm{H}} \subseteq \frac{\mathrm{L}}{\mathrm{H}}$ and $x+\mathrm{H} \notin$ 
$\frac{\mathrm{L}}{\mathrm{H}}$. But $\mathrm{L}$ is J-pure submodule in $\mathrm{M}$, hence by 3, proposition $2.5, \frac{\mathrm{L}}{\mathrm{H}}$ is J-pure submodule in $\frac{\mathrm{M}}{\mathrm{H}}$. Therefore, $\frac{N}{H}$ is a quasi J-pure submodule in $\frac{M}{H}$.

The following proposition gives a characterization of quasi J-pure submodules.

Proposition (2.6)

Let $\mathrm{M}$ be an $\mathrm{R}$ - module and $\mathrm{N}$ be asubmodule of $\mathrm{M}$. Then $\mathrm{N}$ is a quasi J-pure submodule of $\mathrm{M}$ if and only if there exists a collection of submodules $\left\{\mathrm{N}_{\alpha}\right\}_{\alpha \in_{\Lambda}}$, where $\Lambda$ is an index set, such that for each $\alpha \in \Lambda, \mathrm{N}_{\alpha}$ are J-pure submodules of $\mathrm{M}$ and $\mathrm{N}=\mathrm{\cap}_{\alpha \in \Lambda} \mathrm{N}_{\alpha}$.

Proof

Assume that $\mathrm{N}$ is a quasi J-pure submodule of $\mathrm{M}$. If $\mathrm{N}$ is a $\mathrm{J}$-pure submodule of $\mathrm{M}$ then there is nothing to prove. If $\mathrm{N}$ is not $\mathrm{J}$-pure submodule of $\mathrm{M}$, Since $\mathrm{N}$ is quasi $\mathrm{J}$-pure submodule of $\mathrm{M}$, then there exists a collection of J-pure submodules $\left\{\mathrm{N}_{\alpha}\right\}_{\alpha \in \Lambda}$ such that $\mathrm{N} \subseteq \mathrm{\cap}_{\alpha \in \Lambda} \mathrm{N}_{\alpha}$, where $\Lambda$ is an index set. To show that $\cap_{\alpha \in \Lambda} \mathrm{N}_{\alpha} \subseteq \mathrm{N}$, let $x \in \cap_{\alpha \in \Lambda} \mathrm{N}_{\alpha}$, then $x \in \mathrm{N}_{\alpha}$ for each $\alpha \in \Lambda$. Suppose that $x \notin \mathrm{N}$. Since $\mathrm{N}$ is quasi J-pure submodule of $\mathrm{M}$, then $x$ is not contained in any $\mathrm{J}$-pure submodule that contains N. So, $x \notin \mathrm{N}_{\alpha}$, which is a contradiction. Therefore, $x \in \mathrm{N}$ and hence $\mathrm{\cap}_{\alpha \in \Lambda} \mathrm{N}_{\alpha} \subseteq \mathrm{N}$. That is, $\cap_{\alpha \in \Lambda} \mathrm{N}_{\alpha}=\mathrm{N}$.

Conversely, suppose that $\mathrm{N}=\mathrm{n}_{\alpha \in \Lambda} \mathrm{N}_{\alpha}$, where $\mathrm{N}_{\alpha}$ is a J-pure submodule of $\mathrm{M}$ for each $\alpha \in \Lambda$ and $\mathrm{N}_{\alpha}$ containing $\mathrm{N}$. Let $x \in \mathrm{M}$ and $x \notin \mathrm{N}$. Since $\mathrm{N}=\mathrm{n}_{\alpha \in \Lambda} \mathrm{N}_{\alpha}$, so there exists $\beta \in \Lambda$ such that $x \notin \mathrm{N}_{\beta}$. Thus $\mathrm{N} \subseteq \mathrm{N}_{\beta}$ and $x \notin \mathrm{N}_{\beta}$. That is, $\mathrm{N}$ is quasi J-pure submodule in $\mathrm{M}$.

\section{Proposition (2.7)}

Let $M_{1}$ and $M_{2}$ be two R-modules. If $A$ is quasi J-pure submodule of $M_{1}$ and $B$ is quasi J-pure submodule of $M_{2}$, then $A \oplus B$ is quasi J-pure submodule of $M=M_{1} \oplus M_{2}$.

Proof

Let $\left(x_{1}, x_{2}\right) \in \mathrm{M}=\mathrm{M}_{1} \oplus \mathrm{M}_{2}$ with $\left(x_{1}, x_{2}\right) \notin \mathrm{A} \oplus \mathrm{B}$, then either $x_{1} \notin \mathrm{A}$ or $x_{2} \notin \mathrm{B}$. Assume that $x_{1} \notin \mathrm{A}$, since $\mathrm{A}$ is quasi $\mathrm{J}$-pure in $\mathrm{M}$, so there exists a $\mathrm{J}$-pure submodule $\mathrm{L}_{1}$ in $\mathrm{M}_{1}$ such that $\mathrm{L}_{1}$ containing $\mathrm{A}$ and $x_{1} \notin \mathrm{L}_{1}$. But $\mathrm{L}_{1}$ is J-pure in $\mathrm{M}_{1}$, so by 3, proposition 2.6, $\mathrm{L}_{1} \oplus \mathrm{M}_{2}$ is J-pure in $\mathrm{M}$. Also $\mathrm{L}_{1} \oplus \mathrm{M}_{2}$ containing $\mathrm{A} \oplus \mathrm{B}$ and $\left(x_{1}, x_{2}\right) \notin \mathrm{L}_{1} \oplus \mathrm{M}_{2}$.

Similarly, if $x_{2} \notin \mathrm{B}$, then there exists a J-pure submodule in $\mathrm{M}$ containing $\mathrm{A} \oplus \mathrm{B}$ and does not contain $\left(x_{1}, x_{2}\right)$. Therefore, $\mathrm{A} \oplus \mathrm{B}$ is quasi J-pure submodule in $\mathrm{M}$.

The converse of proposition (2.7) is true under certain conditions, as in the following:

\section{Proposition (2.8)}

Let $M_{1}$ and $M_{2}$ be R-modules, $N$ be a submodule in $M_{1}$, and $K$ be a submodule in $M_{2}$ such that $\operatorname{ann}_{R}\left(M_{1}\right)+\operatorname{ann}_{R}\left(M_{2}\right)=R$. If $N \oplus K$ is quasi J-pure submodule in $M=M_{1} \oplus M_{2}$, then $N$ is quasi $J$ pure in $\mathrm{M}_{1}$ and $\mathrm{K}$ is quasi $\mathrm{J}$-pure submodule in $\mathrm{M}_{2}$.

\section{Proof}

To show that $\mathrm{N}$ is quasi $\mathrm{J}$-pure in $\mathrm{M}_{1}$, let $x \in \mathrm{M}_{1}$ and $x \notin \mathrm{N}$. Then $(x, 0) \notin \mathrm{N} \oplus \mathrm{K}$. Since $\mathrm{N} \oplus \mathrm{K}$ is quasi J-pure submodule in $M$, so there exists a J-pure submodule $H$ in $M$ such that $\mathrm{N} \oplus \mathrm{K} \subseteq \mathrm{H}$ and $(x, 0) \notin \mathrm{H}$. Since $\operatorname{ann}_{\mathrm{R}}\left(\mathrm{M}_{1}\right)+\operatorname{ann}_{\mathrm{R}}\left(\mathrm{M}_{2}\right)=\mathrm{R}$, then by a part of the proof of a previous work [6, Proposition (4.2), CH.1], any submodule of $M=M_{1} \oplus M_{2}$ can be written as a direct sum of two submodule of $M_{1}$ and $M_{2}$. Thus $H=A \oplus B$ for some submodules $A$ and $B$ of $M_{1}$ and $M_{2}$, respectively. It follows, by remark and example (2.2) in an earlier study [3], that $\mathrm{A}$ is J-pure submodule in $\mathrm{M}_{1}$ and $\mathrm{B}$ is $\mathrm{J}$-pure submodule in $\mathrm{M}_{2}$. Since $\mathrm{N} \oplus \mathrm{K} \subseteq \mathrm{A} \oplus \mathrm{B}$, so $\mathrm{N} \subseteq \mathrm{A}$ and $\mathrm{K} \subseteq \mathrm{B}$. But $(x, 0) \notin \mathrm{H}=$ $\mathrm{A} \oplus \mathrm{B}$, then $x \in A$. Therefore, $\mathrm{N}$ is $\mathrm{J}$-pure submodule in $\mathrm{M}_{1}$.

Similarly, $\mathrm{K}$ is quasi $\mathrm{J}$-pure submodule in $\mathrm{M}_{1}$.

\section{Remark (2.9)}

The condition $\operatorname{ann}_{R}\left(M_{1}\right)+\operatorname{ann}_{R}\left(M_{2}\right)=R$ is necessary in proposition (2.8). For example, the module $\mathrm{Z}_{8} \oplus \mathrm{Z}_{2}$ is a Z-module. Clearly, $\quad \operatorname{ann}_{\mathrm{z}}\left(\mathrm{Z}_{8}\right)+\operatorname{ann}_{\mathrm{z}}\left(\mathrm{Z}_{2}\right)=2 \mathrm{Z} \neq \mathrm{Z}$. As we have seen in remark and example (2.2), the submodule $\langle(\overline{4}, \overline{0})\rangle=\langle\overline{4}\rangle \oplus\langle\overline{0}\rangle$ is quasi J-pure submodule in $\mathrm{Z}_{8} \oplus \mathrm{Z}_{2}$. But $<\overline{4}>$ is not quasi $\mathrm{J}$-pure submodule in $\mathrm{Z}_{8}$. Because $2 \in \mathrm{Z}_{8}, 2 \notin<\overline{4}>$, there exists no J-pure submodule $\mathrm{L}$ of $\mathrm{Z}_{8}$ containing $\mathrm{N}=\langle\overline{4}\rangle$ and $2 \notin \mathrm{L}$.

Recall that an $R$-module $\mathrm{M}$ is called a multiplication module if for each submodule $\mathrm{N}$ of $\mathrm{M}$ there exists an ideal $I$ of $R$ such that $\mathrm{N}=\mathrm{IM}[7]$. 


\section{Proposition (2.10)}

Let $\mathrm{M}$ be a faithful finitely $\mathrm{J}$-generated multiplication $\mathrm{R}$-module and let $\mathrm{N}$ be a submodule of $\mathrm{M}$. The following statements are equivalent:

(1) $\mathrm{N}$ is a quasi $\mathrm{J}$-pure submodule of $\mathrm{M}$.

(2) $\left[N:_{R} M\right]$ is a quasi $J$-pure ideal of $R$.

\section{Proof}

$(1) \Rightarrow(2)$ Let $\mathrm{r} \in \mathrm{R}$ and $\mathrm{r} \notin\left[\mathrm{N}:_{\mathrm{R}} \mathrm{M}\right]$. Then $r \mathrm{M} \nsubseteq \mathrm{N}$, so there exists $x \in \mathrm{M}$, such that $r x \notin \mathrm{N}$. But $\mathrm{N}$ is a quasi J-pure submodule in $\mathrm{M}$, then there exists a J-pure submodule $\mathrm{K}$ of $\mathrm{M}$ such that $\mathrm{N} \subseteq \mathrm{K}$ and $r x \notin \mathrm{K}$. Since $\mathrm{M}$ is faithful finitely generated $\mathrm{J}$-multiplication, so it is clear that if $\mathrm{K}$ is $\mathrm{J}$-pure submodule in $\mathrm{M}$, then $\left[\mathrm{K}:{ }_{\mathrm{R}} \mathrm{M}\right]$ is $\mathrm{J}$-pure ideal of R. Also $r x \notin \mathrm{K}$ for each $x \in \mathrm{M}$, then $r \notin\left[\mathrm{K}:_{\mathrm{R}} \mathrm{M}\right]$. Hence $\left[\mathrm{K}::_{\mathrm{R}} \mathrm{M}\right]$ is $\mathrm{J}$-pure ideal of $\mathrm{R}$ such that $\left[\mathrm{N}:_{\mathrm{R}} \mathrm{M}\right] \subseteq\left[\mathrm{K}:_{\mathrm{R}} \mathrm{M}\right]$ and $r \notin\left[\mathrm{K}:_{\mathrm{R}} \mathrm{M}\right]$. That is, $\left[\mathrm{N}:_{\mathrm{R}} \mathrm{M}\right]$ is quasi $\mathrm{J}$-pure ideal of $\mathrm{R}$.

\section{Basic Results for Quasi J-regular modules}

In this section, we introduce and study the class of quasi J-regular modules.

\section{Definition (3.1)}

An R-module $\mathrm{M}$ is called quasi J-regular module if every submodule of $\mathrm{M}$ is quasi J-pure.

Recall that an R-module $\mathrm{M}$ is called F-regular if each submodule of $\mathrm{M}$ is pure. Equivalently, an $\mathrm{R}$ module $\mathrm{M}$ is said to be F-regular $\mathrm{R}$ - module if for each $m \in \mathrm{M}, r \in \mathrm{R}$, there exists $t \in \mathrm{R}$ such that $r m=r t r m .[8,9]$

\section{Remarks and Examples (3.2):}

(1) It is clear that every J-regular R-module is quasi J-regular module. But the converse is not true in general.

(2) If $\mathrm{M}$ is J-pure simple R-module, then $\mathrm{M}$ is not quasi J-regular. For example, the Z-modules Q, $\mathrm{Z}_{\mathrm{P} \infty}$.

(3) It is clear that every F-regular R-module is quasi J-regular, but the converse is not true in general. For example, if $\mathrm{M}=\mathrm{Z}_{4}$ as $\mathrm{Z}$-module. $\mathrm{M}$ is quasi J-regular since it is $\mathrm{J}$-regular, but it is not $\mathrm{F}$-regular, by remarks and examples (3.3) in an earlier work [3].

Recall that an $R$-epimorphism $\varphi: M \rightarrow M^{\prime}$ is called small epimorphism if $\operatorname{Ker} \varphi<<M$ [5].

Proposition (3.3)

Let $\mathrm{M}$ be an R-module. Then M is quasi J-regular if and only if $\frac{\mathrm{M}}{\mathrm{N}}$ is quasi J-regular for every small submodule $\mathrm{N}$ of $\mathrm{M}$.

\section{Proof}

Let $\mathrm{N}$ be a small submodule of $\mathrm{M}$ and $\mathrm{K}$ be any submodule of $\mathrm{M}$ containing $\mathrm{N}$. Since $\mathrm{M}$ is quasi $\mathrm{J}$ regular then K is quasi J-pure in M. So, by proposition (2.5), $\frac{K}{N}$ is quasi J-pure in $\frac{M}{N}$. Therefore, $\frac{M}{N}$ is quasi J-regular.

The converse is clear by taking $\mathrm{N}=\langle 0\rangle$.

\section{Corollary (3.4)}

Let $\mathrm{M}$ and $\mathrm{M}^{\prime}$ be two R-modules and $f: \mathrm{M} \longrightarrow \mathrm{M}^{\prime}$ be a small epimorphism. If $\mathrm{M}$ is quasi $\mathrm{J}$ regular, then $\frac{\mathrm{M}}{\mathrm{Kerf}}$ is quasi J-regular.

\section{Proof}

Since $f: \mathrm{M} \longrightarrow \mathrm{M}^{\prime}$ is an epimorphism and $\operatorname{Ker} f<<\mathrm{M}$ and $\mathrm{M}$ are quasi $\mathrm{J}$-regular, then $\frac{\mathrm{M}}{\operatorname{Kerf}}$ is quasi J-regular by proposition (3.3).

Recall that a non-zero R-module $\mathrm{M}$ is called a hollow if every proper submodule of $\mathrm{M}$ is a small [10].

\section{Corollary (3.5)}

Let $\mathrm{M}$ be a hollow R-module and $\mathrm{M}^{\prime}$ be any $\mathrm{R}$-module. If $f: \mathrm{M} \longrightarrow \mathrm{M}^{\prime}$ is an epimorphism, then $\frac{\mathrm{M}}{\text { Kerf }}$ is quasi J-regular.

Proof: It is clear.

Corollary (3.6)

Let $M_{1}$ and $M_{2}$ be R-modules such that $\operatorname{ann}_{R}\left(M_{1}\right)+\operatorname{ann}_{R}\left(M_{2}\right)=R$. Then $M=M_{1} \oplus M_{2}$ is quasi $\mathrm{J}$-regular if and only if $\mathrm{M}_{1}$ and $\mathrm{M}_{2}$ are quasi J-regular. 


\section{Proof}

Assume that $M=M_{1} \oplus M_{2}$ is quasi J-regular and let $N_{1}$ be a submodule of $M_{1}$, then we have to show that $\mathrm{N}_{1}$ is quasi J-pure in $\mathrm{M}_{1}$. Let $x \in \mathrm{M}_{1}$ and $x \notin \mathrm{N}_{1}$, then there exists $\mathrm{N}_{1} \subseteq \mathrm{L}_{1}$ such that $x \notin \mathrm{L}_{1} .(x, 0) \in \mathrm{M}=\mathrm{M}_{1} \oplus \mathrm{M}_{2},(x, 0) \notin \mathrm{N}=\mathrm{N}_{1} \oplus \mathrm{N}_{2}$. Since $\mathrm{M}$ is quasi J-regular, then $\mathrm{N}=\mathrm{N}_{1} \oplus \mathrm{N}_{2}$ is quasi J-pure. Then there exists a J-pure submodule $\mathrm{L}$ of $\mathrm{M}$ such that $\mathrm{N} \subseteq \mathrm{L}$ and $(x, 0) \notin \mathrm{L}$. Since $\operatorname{ann}_{\mathrm{R}}\left(\mathrm{M}_{1}\right)+\operatorname{ann}_{\mathrm{R}}\left(\mathrm{M}_{2}\right)=\mathrm{R}$, then $\mathrm{L}=\mathrm{L}_{1} \oplus \mathrm{L}_{2}$ where $\mathrm{L}_{1}$ and $\mathrm{L}_{2}$ are submodules in $\mathrm{M}_{1}$ and $\mathrm{M}_{2}$, respectively, by remarks and examples (2.2) in the above mentioned study [3]. $\mathrm{N}_{1} \subseteq$ $\mathrm{L}_{1}$ and $x \notin \mathrm{L}_{1}$. Thus $\mathrm{M}_{1}$ is quasi J-regular.

Similarly $\mathrm{M}_{2}$ is quasi J-regular.

Conversely, assume that $\mathrm{M}_{1}$ and $\mathrm{M}_{2}$ are quasi J-regular and $\mathrm{M}=\mathrm{M}_{1} \oplus \mathrm{M}_{2}$. Let $\mathrm{N}$ be a submodule of $M=M_{1} \oplus M_{2}$. Since $\operatorname{ann}_{R}\left(M_{1}\right)+\operatorname{ann}_{R}\left(M_{2}\right)=R$, then $N=N_{1} \oplus N_{2}$ where $N_{1}$ is a submodule in $\mathrm{M}_{1}$ and $\mathrm{N}_{2}$ is a submodule of $\mathrm{M}_{2}$. Since $\mathrm{M}_{1}$ and $\mathrm{M}_{2}$ are quasi J-regular, then $\mathrm{N}_{1}$ and $\mathrm{N}_{2}$ are quasi Jpure, hence by proposition (2.8), $\mathrm{N}_{1} \oplus \mathrm{N}_{2}$ is quasi J-pure. Therefore, $\mathrm{N}=\mathrm{N}_{1} \oplus \mathrm{N}_{2}$ is quasi J-pure. Thus $\mathrm{M}$ is quasi J-regular.

Recall that a ring $\mathrm{R}$ is called a quasi -regular ring if every ideal in $\mathrm{R}$ is quasi -pure [4].

Definition (3.7)

Let $\mathrm{R}$ be a ring, then $\mathrm{R}$ is called a quasi $\mathrm{J}$-regular ring if every ideal in $\mathrm{R}$ is quasi $\mathrm{J}$-pure.

\section{Remarks and Examples (3.8)}

(1) It is clear that every J-regular ring is quasi J-regular ring. But the converse is not true in general. We have no example.

(2) Every regular ring is quasi J-regular, but the converse is not true in general. For example, $\mathrm{Z}_{9}$ is quasi J-regular ring but not regular.

(3) Let $\mathrm{R}$ be an integral domain. If $\mathrm{R}$ is quasi J-regular, then $\mathrm{R}$ is a field.

\section{Proof}

Since $\mathrm{R}$ is quasi J-regular, then every ideal $\mathrm{I}$ of $\mathrm{R}$ is quasi J-pure. So by proposition (2.6), there exists a collection of J-pure ideals $\left\{I_{\alpha}\right\}_{\alpha \in \Lambda}$ where $\Lambda$ is some index set, such that $I=\cap_{\alpha \in \Lambda} I_{\alpha}$. Since $R$ is integral domain, so by a previous study [11, proposition 2.5], $\mathrm{R}$ is J-pure simple. Thus $\mathrm{R}$ has no J-pure ideals, except $<0>$ and R. That is, $\cap_{\alpha \in \Lambda} \mathrm{I}_{\alpha}=<0>$ or R, and hence $\mathrm{I}=<0>$ or $\mathrm{I}=\mathrm{R}$. Therefore, $\mathrm{R}$ is a field.

Recall that a submodule $N$ of an $R$ - module $M$ is called a maximal submodule of $M$, if whenever $K$ is a submodule of $M$ with $K \supsetneq N$, then $K=M$ [5].

(4) If R is quasi $\mathrm{J}$-regular ring, then every prime ideal of R that contain in JI where JI intersection of all maximal ideals.

Proof

Let $\mathrm{P}$ be a prime ideal in the ring $\mathrm{R}$. Since $\mathrm{R}$ is quasi $\mathrm{J}$-regular and $\mathrm{P} \subseteq \mathrm{J}(R)$, then by a previous study [11, proposition 2.5], $\frac{R}{P}$ is quasi J-regular. But $\frac{R}{P}$ is an integral domain, thus $\frac{R}{P}$ is a field by the above remark (3). Therefore, $\mathrm{P}$ is maximal.

(5) Let $\mathrm{R}$ be a quasi J-regular ring. If $\mathrm{JI}=\langle 0\rangle$, then $\mathrm{R}$ is regular.

\section{Proof}

Since R is quasi J-regular, then by remark (4), every prime ideal of $\mathrm{R}$ is maximal. But $\mathrm{J} \mathrm{I}=\langle 0\rangle$, so by an earlier work [12], $\mathrm{R}$ is regular.

Recall that a proper ideal $I$ of a ring $R$ is said to be a prime ideal if for each $a, b \in R$ such that $a . b \in$ $I$, then either $a \in I$ or $b \in I[5]$.

(6) Every quasi J-regular ring is nearly regular, where a ring $R$ is called nearly ring if $R / J I$ is regular ring [13].

\section{Proof}

Let $\mathrm{R}$ be a quasi J-regular ring. Then $\mathrm{R} / \mathrm{JI}$ is quasi $\mathrm{J}$-regular by the above mentioned study [11, proposition 2.5]. So by the above remark (4), every prime ideal of $R / J I$ is maximal ideal, and since $J(R / J(R))=0$, therefore by an earlier work [13], R/JI is regular. Thus $\mathrm{R}$ is nearly regular.

(7) The converse of Remark (6) is not true in general. For example, the $\mathrm{Z}$-module $\mathrm{Z}_{8}$ is nearly regular but not quasi J-regular ring. 


\section{Theorem (3.9)}

Let $\mathrm{M}$ be a faithful finitely generated multiplication $\mathrm{R}$-module. Then $\mathrm{M}$ is quasi J-regular module if and only if $\mathrm{R}$ is quasi J-regular ring.

\section{Proof}

Let $\mathrm{N}$ be a submodule of $\mathrm{M}$. Since $\mathrm{M}$ is a multiplication R-module, then $\mathrm{N}=\mathrm{I} \mathrm{M}$ for some ideal in R. Since R is quasi J-regular, then $I=\cap_{\alpha \in \Lambda} I_{\alpha}$ by Proposition (2.6), where $I_{\alpha}$ is a J-pure ideal of R containing I. Thus $N=\left(\cap_{\alpha \in \Lambda} I_{\alpha}\right) M$. Since $M$ is faithful multiplication, then $\left(\cap_{\alpha \in \Lambda} I_{\alpha}\right) M=$ $\mathrm{n}_{\alpha \in \Lambda}\left(\mathrm{I}_{\alpha} \mathrm{M}\right)_{\alpha}[14]$.

Claim: I M is J-pure in M. Since I is J-pure in R, then

$$
\begin{aligned}
& \mathrm{IJ}(\mathrm{M}) \cap \mathrm{N}=\mathrm{IJ}(\mathrm{M}) \cap \mathrm{K} \mathrm{M} \text { for some ideal } \mathrm{K} \text { of } \mathrm{R} \\
& =(\mathrm{I} \cap \mathrm{K}) \mathrm{M} \quad \text { since } \mathrm{M} \text { is faithful multiplication. } \\
& =(\mathrm{I} \mathrm{K}) \mathrm{M} \quad \text { since } \mathrm{R} \text { is quasi J-regular ring. } \\
& \mathrm{I} J(\mathrm{M}) \cap \mathrm{N}=\mathrm{I} N \\
& =\mathrm{I}(\mathrm{K} \mathrm{M})
\end{aligned}
$$

Thus $\mathrm{N}=\mathrm{K} \mathrm{M}$ is $\mathrm{J}$-pure in $\mathrm{M}$ and $\mathrm{M}$ is quasi $\mathrm{J}$-regular.

Conversely, let I be an ideal of the ring R. We have to show that I is quasi J-pure. I M is a submodule of M. Since M is quasi J-regular, then by proposition (2.6) I $M=\cap_{\alpha \in \Lambda} L_{\alpha}$ where $L_{\alpha}$ is Jpure submodules of $M$ containing I $M$ for each $\alpha \in \Lambda$. Put $L_{\alpha}=I_{\alpha} M$. Thus $I M=\cap_{\alpha \in \Lambda} L_{\alpha}=$ $\cap_{\alpha \in \Lambda} I_{\alpha} M$, since $M$ is faithful finitely generated, by the $\frac{1}{2}$ cancellation property [12]. Then, $I=\cap_{\alpha \in \Lambda} I_{\alpha}$. Claim: $\mathrm{I}_{\alpha}$ is J-pure in $\mathrm{R}$ and $\mathrm{I} \subseteq \mathrm{I}_{\alpha}$. Let $\mathrm{K} \subsetneq \mathrm{J}(R)$ be an ideal of $\mathrm{R}$.

$$
\begin{aligned}
\left(I_{\alpha} \cap K\right) M & =I_{\alpha} M \cap K M \quad \text { Since } M \text { is faithful multiplication. } \\
& =L_{\alpha} \cap K M \\
\left(I_{\alpha} \cap K\right) M & =I_{\alpha} K M
\end{aligned}
$$

Thus $\mathrm{I}_{\alpha} \cap \mathrm{K}=\mathrm{I}_{\alpha} \mathrm{K}$ [8], which implies that $\mathrm{I}_{\alpha}$ is J-pure in R. Also, since $\mathrm{I}_{\alpha} \mathrm{M}=\mathrm{IM} \subseteq \mathrm{L}_{\alpha}$, thus $\mathrm{I} \subseteq$ $\mathrm{I}_{\alpha}$, so $\mathrm{R}$ is quasi J-regular.

\section{References}

1. Anderson, E.W. and K.R. 1992. "Ring and categories of module", Spring- New York.

2. Fieldhouse, D.J 1969. "Pure Theories", h. Ann. 184: 1-18.

3. Shaibani, R. M. and AL-Mothafar, N. S. 2019. "Properties of J-Regular modules",Iraqi Journal of Science, 60(3): 647-652. DOI: 10.24996/ijs. 60.3.23.

4. AL- Mothafar, N.S. 2002. "Sums and Intersection of Submodules", Ph.D. Thesis University of Baghdad, Baghdad, Iraq.

5. Kasch, F. 1982. "Modules and Rings," London Mathematical Society Monographs, Vol.17, New York; Academic Press.

6. Abbas, M.S. 1991. "On fully stable module", Ph.D. Thesis, University of Baghdad, Baghdad, Iraq.

7. Smith, P.F.1981. "Some Remarks on Multiplication Modules", Arch Math. 50,223-235.

8. Inaam M.A. H. 2013. "Coregular Modules", Iraqi Journal of Science. 54(3): 836-841.

9. Chatham T.J and Enoch's E.E. 1981."Regular modules", Math. Japonica, 26: 9-12

10. Fieldhouse D.J. 1970. Pure Simple and Indecomposable Ring, Can. Math. Bull. 13: 77-78.

11. 11. Shaibani, R. M. and AL-Mothafar, N. S. 2019." Modules With The J-Pure Intersection (Sum) Property", There is appear.

12. AL-Baharaany, B.H. 2000. Module with the Pure Intersection Property, Ph.D. Thesis, University of Baghdad, Baghdad, Iraq.

13. EI-Bast Z.A. and Smith, P.F. 1988. Multiplication Modules, Comm. Algebra, 16(4): 775-779.

14. Ansari H. Toroghy and Farshadifar. 2009."Strongly Comultiplication Modules", CMUJ.Nat.Sci. 8(1): 105-113. 